Journal of Inorganic Biochemistry

\title{
Ruffling is Essential for Staphylococcus aureus IsdG-catalyzed Degradation of Heme to Staphylobilin
}

\author{
Ariel E. Schuelke-Sanchez, Amanda R. Cornetta, Taylor A. J. Kocian, Matthew A. Conger, and Matthew D. \\ Liptak*
}

Department of Chemistry, University of Vermont, Burlington, VT 05405, United States of America

\section{ARTICLE INFO}

Keywords:

Biliverdin

Enzyme Kinetics

Heme Oxygenase

Magnetic Circular Dichroism

Nuclear Magnetic Resonance

Staphylobilin
ABSTRACT

Non-canonical heme oxygenases are enzymes that degrade heme to non-biliverdin products within bacterial heme iron acquisition pathways. These enzymes all contain a conserved second-sphere Trp residue that is essential for enzymatic turnover. Previous studies have revealed several important roles for the conserved second-sphere Trp in Staphylococcus aureus IsdG, S. aureus IsdI, and Mycobacterium tuberculosis MhuD. However, a general model for the geometric, electronic, and functional role of the second-sphere Trp had not been deduced prior to this work. Here, UV/Vis absorption (Abs) and circular dichroism (CD) spectroscopies were employed to show that the W67F variant of IsdG perturbs the heme substrate conformation without altering the protein secondary structure. In general, it can now be stated that a dynamic equilibrium between "planar" and "ruffled" substrate conformations exists within non-canonical heme oxygenases, and that the second-sphere Trp favors population of the "ruffled" substrate conformation. ${ }^{1} \mathrm{H}$ nuclear magnetic resonance and magnetic CD spectroscopies were used to characterize the electronic structures of IsdG and IsdI variants with different substrate conformational distributions. These data revealed that the "ruffled" substrate conformation promotes partial porphyrin-to-iron electron transfer, which makes the meso carbons of the porphyrin ring susceptible to radical attack. Finally, UV/Vis Abs spectroscopy was utilized to quantify the enzymatic rates, and electrospray ionization mass spectrometry was used to identify the product distributions, for variants of IsdG with altered substrate conformational distributions. In general, the rate of heme oxygenation by non-canonical heme oxygenases depends upon the population of the "ruffled" substrate conformation. Also, the production of staphylobilin or mycobilin by these enzymes is correlated with the population of the "ruffled" substrate conformation, since variants that favor population of the "planar" substrate conformation yield significant amounts of biliverdin. These data can be understood within the framework of a concerted rearrangement mechanism for the monooxygenation of heme to meso-hydroxyheme by non-canonical heme oxygenases. However, the mechanisms of IsdG/IsdI and MhuD must diverge following this intermediate in order to generate distinct staphylobilin and mycobilin products, respectively.

\section{Introduction}

Non-canonical heme oxygenases exhibit remarkable relationships between substrate structure, electronic structure, and enzyme function. When the X-ray crystal structure of cyanide-inhibited Staphylococcus aureus IsdI (IsdI-heme$\mathrm{CN}$ ) was reported in 2011 [1], it represented the most ruffled protein-bound heme reported. It was later shown that this ruffling, a $b_{1 u}$ out-of-plane distortion from idealized $D_{4 h}$ symmetry [2], depended upon a second-sphere interaction between Trp66 and the heme substrate [3]. A few years later, X-ray crystallography revealed that a second non-canonical heme oxygenase, Mycobacterium tuberculosis MhuD, also induces a significant ruffling deformation of its heme substrate, albeit to a lesser extent [4]. The heme substrate ruffling by Mhu also depends upon a steric interaction with a second-sphere Trp residue, Trp66 [5]. Furthermore, detailed spectroscopic and computational studies of several MhuD variants demonstrated that the heme distortion detected by X-ray crystallography is the weighted average of a dynamic equilibrium between two distinct heme substrate conformations: "planar" and "ruffled" [6]. Most recently, spectroscopically-validated hybrid quantum mechanics/molecular mechanics (QM/MM) calculations suggested that $S$. aureus IsdG, a third non-canonical heme oxygenase, induces an intermediate degree of heme ruffling compared to IsdI and MhuD (Fig. 1) [7]. The observation of two distinct heme conformations within the MhuD active

\footnotetext{
* Corresponding author

E-mail address: matthew.liptak@uvm.edu (M.D. Liptak)
}

site is fairly unique within the heme protein literature, and the possibility that IsdG and IsdI also stabilize two distinct heme substrate conformations had not been evaluated prior to this work.

The highly ruffled heme substrate of non-canonical heme oxygenases is responsible for unique electronic structures that promote novel biochemical reactivity. Characterization of IsdI-heme-CN with ${ }^{1} \mathrm{H}$ nuclear magnetic resonance (NMR) spectroscopy revealed that this species has an unusual ${ }^{2} B_{2 g}$ electronic ground state with an $\mathrm{Fe}\left(3 d_{\mathrm{xz}, \mathrm{yz}}\right)^{4}\left(3 d_{\mathrm{xy}}\right)^{1}$ electron configuration [1], which had previously only been observed for highly ruffled model complexes [8]. Preparation and ${ }^{1} \mathrm{H}$ NMR characterization of W66Y IsdI-heme-CN, which houses a less ruffled heme substrate, clarified that the unusual ${ }^{2} B_{2 g}$ electronic ground state of WT IsdI-heme-CN is derived from the large ruffling deformation of heme [3]. Subsequent characterization of cyanide-inhibited MhuD (MhuD-heme-CN) using magnetic circular dichroism (MCD) spectroscopy demonstrated that this species also has the unusual ${ }^{2} B_{2 g}$ electronic ground state, and hinted at underlying complexity [4]. Indeed, spectroscopic characterization of additional MhuD variants, coupled with multi-reference perturbation theory calculations, revealed that MhuD-heme-CN has a ${ }^{2} B_{2 g}$ electronic ground state corresponding to the "ruffled" conformation with a lowlying ${ }^{2} E_{g}$ excited state associated with the "planar" substrate conformation [6]. The ${ }^{2} E_{g}$ electronic state has an $\mathrm{Fe}\left(3 d_{\mathrm{xy}}\right)^{2}\left(3 d_{\mathrm{xz}, \mathrm{yz}}\right)^{3}$ electron configuration, and is the most typical electronic ground state for low-spin ferric heme [9]. Spectroscopic characterization of cyanide-inhibited IsdG (IsdG-heme-CN) has 


\begin{tabular}{|ll|}
\hline Abbreviations & \\
Abs & absorption \\
$\mathrm{CD}$ & circular dichroism \\
DSS & sodium 2,2-dimethyl-2-silapentane-5-sulfonate \\
ESI-MS & electrospray ionization mass spectrometry \\
FPLC & fast protein liquid chromatography \\
IsdG-heme & heme-bound IsdG \\
IsdG-heme-CN & cyanide-inhibited IsdG \\
IsdI-heme & heme-bound IsdI \\
IsdI-heme-CN & cyanide-inhibited IsdI \\
KP & potassium phosphate \\
MCD & magnetic circular dichroism \\
MeCN & acetonitrile \\
MhuD-heme-CN & cyanide-inhibited MhuD \\
MS & mass spectrometry \\
NaP & sodium phosphate \\
NMR & nuclear magnetic resonance \\
QM/MM & quantum mechanics / molecular mechanics \\
TEV & tobacco etch virus \\
VTVH & variable-temperature, variable-field \\
\hline
\end{tabular}

also provided evidence for a thermal mixture of ${ }^{2} B_{2 g}$ and ${ }^{2} E_{g}$ electronic states (Fig. 1) [10, 11]. These electronic structure changes assume additional significance in light of the fact that the electronic structure change induced by the "ruffled" conformation, partial porphyrin to iron electron transfer, promotes porphyrin hydroxylation [12].

Perhaps not surprisingly, the unusual geometric and electronic structures of the heme substrate in non-canonical heme oxygenases have often been invoked to explain the novel mechanisms of these enzymes. Prior to the characterizations of the geometric and electronic structures of S. aureus IsdG and IsdI described above, it was discovered that these enzymes degrade heme to novel staphylobilin products that are distinct from the biliverdin products of canonical heme oxygenases [13]. Subsequent studies identified formaldehyde as the $\mathrm{C} 1$ product [14], and identified two reaction intermediates: mesohydroxyheme and formyloxobilin [15]. A concerted rearrangement mechanism has been proposed for the initial monooxygenation of heme to mesohydroxyheme $[10,16]$, but the remaining mechanistic steps from mesohydroxyheme to staphylobilin remain unknown with minimal chemical precedent to inform reasonable hypotheses. It turns out that MhuD degrades heme to a third product, mycobilin [17], via sequential monooxygenation and dioxygenation steps [18]. Recently, it has been reported that both the R26S and W66F second-sphere substitutions can change the primary MhuD product from mycobilin to biliverdin $[19,20]$. These observations have been attributed to a mechanism where the structural and electronic dynamics of heme-bound MhuD are critical: MhuD converts "ruffled" heme to meso-hydroxyheme and "planar" meso-hydroxyheme to mycobilin. The proposed monooxygenation mechanism is consistent with a recent $\mathrm{QM} / \mathrm{MM}$ study of MhuD-catalyzed heme degradation [21]. In this work, we sought to determine whether the structural and electronic dynamics observed for MhuD are also present in IsdG and IsdI, as well as whether they can be exploited to alter the enzymatic product of IsdG.

This article elucidates the influence of the Trp67 residue on the structure, electronic structure, and mechanism of S. aureus IsdG. The W67F variant was designed with the goal of isolating the contribution of the second-sphere Trp residue to the structure and electronic structure of the IsdG-bound heme substrate. In order to separate the contributions of the amino acid substitution to the substrate and protein structures, circular dichroism (CD) spectroscopy was used to compare the secondary structures of WT and W67F IsdG. A recently established UV/Vis absorption (Abs) spectroscopy-based assay was employed to quantify heme ruffling instead of the more laborious crystallography-based approach previously utilized for IsdI [1, 3, 5]. Rufflinginduced heme electronic structure changes have been invoked as a key component in non-canonical heme oxygenase mechanisms [10, 14, 16, 18, 20, 21], so a careful assessment of the heme electronic structure of these variants using ${ }^{1} \mathrm{H}$ NMR and MCD spectroscopies was carried out. Finally, the rates of heme oxygenation by WT and W67F IsdG were quantified using a UV/Vis Absbased assay and the products of these enzyme variants were identified using electrospray ionization mass spectrometry (ESI-MS) [15]. The implications of these data in terms of a general model for the role of a conserved second-sphere Trp in non-canonical heme oxygenases will be discussed.

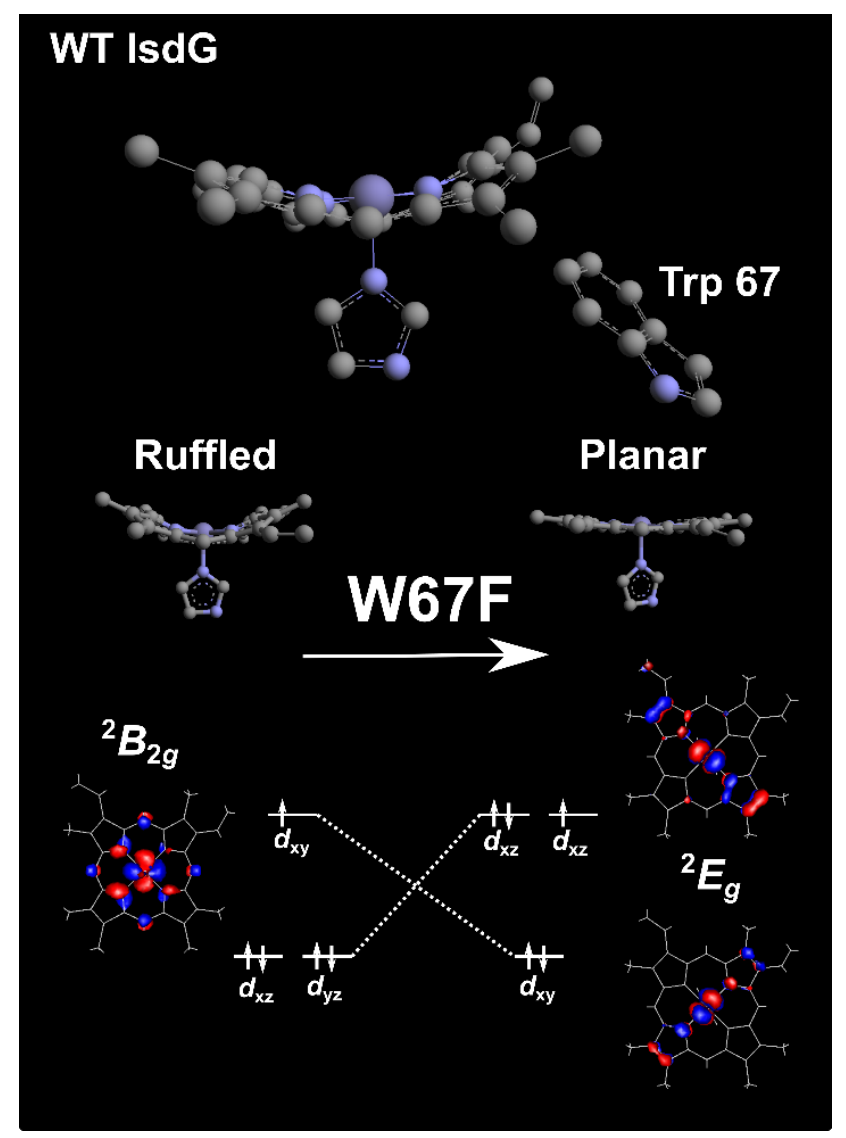

Fig. 1. WT IsdG induces an out-of-plane ruffling distortion of its heme substrate based upon spectroscopically-validated QM/MM calculations (top) [7]. The W67F substitution was hypothesized to decrease heme ruffling by reducing steric interactions between residue 67 and the substrate (middle). This heme structural change would be expected to change the ground state electronic configuration from ${ }^{2} B_{2 g}$ to ${ }^{2} E_{g}$ by decreasing mixing of the $\mathrm{Fe} 3 d_{\mathrm{xy}}$ and porphyrin $a_{2 u}$ orbitals (bottom) resulting in altered heme oxygenation activity $[5,20]$.

\section{Experimental}

Unless noted otherwise, all materials in this work were purchased from Fisher Scientific and used without further purification.

\subsection{Protein expression and purification}

Single amino acid substitutions were introduced into genes encoding $S$. aureus IsdG and IsdI using site-directed mutagenesis. The preparations of a pET15b ( $A m p^{\mathrm{r}}$, Novagen) vector encoding a short-linker construct of WT IsdG [22], a pET15b (Amp ${ }^{r}$, Novagen) vector encoding WT IsdI [6], and a PRK793 $\left(\mathrm{Amp}^{r}\right.$ ) vector encoding S219V tobacco etch virus (TEV) protease have been previously described [23]. The W67F mutation was introduced into the recombinant isdG gene, and the W66Y mutation was introduced into the isdI gene, using the QuikChange Lightning site-directed mutagenesis kit (Agilent). 
DNA primers for the isdG mutagenesis reaction were designed according to the QuikChange manual and obtained from Midland Certified Reagent Company (Table S1). DNA primers for isdI mutagenesis were obtained from Integrated DNA Technologies (Table S2). DNA sequencing performed by the University of Vermont DNA analysis facility confirmed successful preparations of W67F IsdG and W66Y IsdI (Tables S3-S4).

WT IsdG, W67F IsdG, and W66Y IsdI were expressed as previously reported for short-linker WT IsdG [7]. WT IsdG and W67F IsdG were purified using nickel affinity chromatography as previously described for short-linker WT IsdG, but some minor modifications to the chromatography procedure were required to obtain pure W66Y IsdI. Specifically, the nickel-charged HiTrap Chelating HP column (GE Healthcare) equilibrated with $50 \mathrm{mM}$ Tris $\mathrm{pH}$ 7.4, $150 \mathrm{mM} \mathrm{NaCl}$ was washed with a linear gradient of imidazole from 0 to 150 $\mathrm{mM}$ prior to elution of W66Y IsdI with $300 \mathrm{mM}$ imidazole using an Äkta Pure fast protein liquid chromatography (FPLC) system (GE Healthcare). The Nterminal $\mathrm{His}_{6}$-tags were removed as previously described for WT IsdI [6, 24]. W67F IsdG and W66Y IsdI were obtained in $>99 \%$ and $>95 \%$ purity, respectively, as determined by SDS-PAGE gel electrophoresis (Figs. S1-S2). Apoprotein concentrations were determined by Bradford assay with bovine serum albumin (Pierce) as the standard.

The secondary structures of WT and W67F IsdG were assessed using CD spectroscopy. The CD spectrum of WT IsdG was reported previously [7]. W67F IsdG was exchanged into $10 \mathrm{mM}$ potassium phosphate $\left(\mathrm{KP}_{\mathrm{i}}\right) \mathrm{pH} 7.4$ using a PD-10 desalting column and loaded into a $2 \mathrm{~mm}$ path length quartz cuvette (Starna). CD data were acquired from 260 to $190 \mathrm{~nm}$ with a $20 \mathrm{~nm} / \mathrm{min}$ scan speed, a $1.0 \mathrm{~nm}$ bandwidth, an $8 \mathrm{~s}$ digital integration time, and a $0.5 \mathrm{~nm}$ data pitch using a Jasco J-1700 spectrophotometer. The CD spectra were analyzed using the Dichroweb program SELCON3 with reference dataset 4 [25-29].

\subsection{Spectroscopic characterization}

The heme-bound and cyanide-inhibited forms of IsdG and IsdI were characterized with UV/Vis Abs spectroscopy as described previously [1, 30]. Briefly, heme-bound forms of IsdG (IsdG-heme) and IsdI (IsdI-heme) were prepared in $50 \mathrm{mM}$ Tris $\mathrm{pH} 7.4,150 \mathrm{mM} \mathrm{NaCl}$ as described previously for WT IsdG-heme [7, 30]. The extinction coefficients of W67F IsdG-heme and W66Y IsdI-heme were determined to be $\varepsilon_{412}=117.1 \mathrm{mM}^{-1} \mathrm{~cm}^{-1}$ and $\varepsilon_{401}=92.4 \mathrm{mM}^{-}$ ${ }^{1} \mathrm{~cm}^{-1}$, respectively, by employing the pyridine hemochrome assay [31]. In order to determine the extinction coefficients of IsdG-heme-CN and IsdI-heme-CN, a single crystal of $\mathrm{KCN}$ was added to a heme-bound enzyme sample of known concentration as described previously for IsdG-heme- $\mathrm{CN}$ [10]. The extinction coefficients of WT IsdG-heme-CN, W67F IsdG-heme-CN, and W66Y IsdIheme-CN were determined to be $\varepsilon_{420}=94.9 \mathrm{mM}^{-1} \mathrm{~cm}^{-1}, \varepsilon_{418}=95.8 \mathrm{mM}^{-1} \mathrm{~cm}^{-1}$, and $\varepsilon_{416}=94.4 \mathrm{mM}^{-1} \mathrm{~cm}^{-1}$, respectively. UV/Vis Abs data were acquired from 700 to $300 \mathrm{~nm}$ on a Cary 100 spectrophotometer with a scan rate of $600 \mathrm{~nm} / \mathrm{min}$, $2 \mathrm{~nm}$ bandwidth, $0.1 \mathrm{~s}$ digital integration time, and $1 \mathrm{~nm}$ data interval.

The ground state electronic structure of $\mathrm{IsdG}-$ heme- $\mathrm{CN}$ was characterized using ${ }^{1} \mathrm{H}$ NMR spectroscopy in a similar manner to that previously described for IsdI-heme-CN [1]. WT and W67F IsdG-heme-CN were prepared as described above for UV/Vis Abs characterization, exchanged into sodium phosphate $\left(\mathrm{NaP}_{\mathrm{i}}\right) \mathrm{pH} 7.4$, concentrated using an Amicon stirred cell (Millipore), and diluted with $\mathrm{D}_{2} \mathrm{O}$ (Cambrdige Isotope Laboratories). This procedure yielded 0.84 and $1.58 \mathrm{mM}$ samples of WT and W67F IsdG-heme-CN, respectively, in $10 \mathrm{mM} \mathrm{NaP}_{\mathrm{i}} \mathrm{pH} 7.4,10 \% \mathrm{D}_{2} \mathrm{O}(v / v) .{ }^{1} \mathrm{H} \mathrm{NMR}$ data was acquired on a Varian Unity Inova $500 \mathrm{MHz}$ spectrometer equipped with an inverse tripleresonance probe. 256 scans were acquired with a $1.5 \mathrm{~s}$ relaxation delay, presaturation of the ${ }^{1} \mathrm{H}_{2} \mathrm{O}$ resonance during this delay, a $1 \mathrm{~s}$ acquisition time, and a $30 \mathrm{kHz}$ sweep width at $25{ }^{\circ} \mathrm{C}$. The ${ }^{1} \mathrm{H}$ NMR spectra were indirectly referenced to sodium 2,2-dimethyl-2-silapentane-5-sulfonate (DSS, Cambridge Isotope Laboratories) via the residual ${ }^{1} \mathrm{H}_{2} \mathrm{O}$ resonance and processed using the
ACD/Labs NMR processor with $10 \mathrm{~Hz}$ exponential line broadening and zero filling to 32786 points.

The excited state electronic structures of IsdG-heme-CN and IsdI-heme$\mathrm{CN}$ were interrogated using MCD spectroscopy. Samples of W67F IsdGheme-CN and W66Y IsdI-heme-CN in $50 \mathrm{mM} \mathrm{KP}$ i pH 7.4, $60 \%$ glycerol $(v / v)$ were prepared as described previously for IsdI-heme-CN [6]. MCD spectral data were acquired using a home-built setup consisting of either a Jasco J-815 (IsdG-heme-CN) or Jasco J-1700 (IsdI-heme-CN) spectrometer in conjunction with an Oxford SM4000-8T Spectromag. MCD data was collected with a scanning speed of $200 \mathrm{~nm} / \mathrm{min}$, a bandwidth of $1 \mathrm{~nm}$, a digital integration time of $0.25 \mathrm{~s}$, and a data pitch of $0.5 \mathrm{~nm}$. Data was acquired for temperatures ranging from 2 to $10 \mathrm{~K}$ and magnetic fields ranging from -7 to $+7 \mathrm{~T}$. In addition, variable-temperature, variable-field (VTVH) saturation magnetization curves were measured ranging from 2 to $20 \mathrm{~K}$ with a magnetic field ramp of $0.7 \mathrm{~T} / \mathrm{min}$ between $\pm 7 \mathrm{~T}$ for the 424 and $422 \mathrm{~nm}$ bands of W67F IsdG-heme- $\mathrm{CN}$ and W66Y IsdI-heme-CN, respectively. For all MCD data, the CD contribution was eliminated by subtracting the negative field data from the positive field data and dividing by 2 .

\subsection{Enzyme activity assays}

The enzymatic activities of WT and W67F IsdG were assayed in a manner similar to that previously described for WT enzyme $[13,30]$. Briefly, WT and W67F IsdG-heme were prepared as described above for UV/Vis Abs spectroscopic characterization. $10 \mu \mathrm{M}$ IsdG-heme was mixed with $10 \mathrm{mM}$ sodium ascorbate, 200 units of bovine liver catalase (Sigma Aldrich), and $1 \mathrm{mM}$ ethylenediaminetetraacetic acid in $50 \mathrm{mM} \mathrm{KP} \mathrm{i}_{\mathrm{i}} \mathrm{pH}$ 7.4. UV/Vis Abs spectra of the reaction mixture were acquired every $5 \mathrm{~min}$ over a course of $60 \mathrm{~min}$ on a Cary 100 spectrophotometer using the instrumental parameters listed above. An additional assay was completed for W67F IsdG with $30 \mu \mathrm{M}$ IsdG-heme, $1 \mathrm{mM}$ sodium ascorbate, 200 units of bovine liver catalase (Sigma Aldrich), and $1 \mathrm{mM}$ ethylenediaminetetraacetic acid in $50 \mathrm{mM} \mathrm{KP}$ i $\mathrm{pH}$ 7.4. This additional assay was monitored by UV/Vis Abs spectroscopy for 120 minutes using the parameters noted above.

The products of WT and W67F IsdG-catalyzed heme degradation were identified using in proteo mass spectrometery (MS) according to a procedure similar to that previously described for WT enzyme [15]. After $60 \mathrm{~min}$, the IsdG-heme reaction mixtures described above were loaded onto a $\mathrm{C}_{18}$ column equilibrated with $2 \%$ acetonitrile $(\mathrm{MeCN})$ with $0.1 \%$ formic acid $(v / v)$ in water $(v / v)$ using a Shimadzu Prominence high-performance liquid chromatography system. Tetrapyrroles were eluted with a linear gradient from $2 \%$ to $98 \% \mathrm{MeCN}$ with $0.1 \%$ formic acid $(v / v)$ in water $(v / v)$ over $50 \mathrm{~min}$. ESI-MS data for $\mathrm{m} / \mathrm{z}$ 600-1500 was acquired in positive ion mode using an ABI Sciex 4000 QTrap Pro hybrid triple-quadrupole/linear ion trap.

\section{Results}

\subsection{The W67F substitution does not perturb the secondary structure of IsdG}

Analyses of X-ray crystal structure and UV CD data validated usage of the SELCON3 algorithm to extract secondary structure information from the $\mathrm{CD}$ spectra of IsdG variants. UV CD spectra of proteins contain valuable information regarding secondary structure since this region of the spectrum is dominated by electronic transitions within the amide backbone groups, but a suitable analysis algorithm is needed to extract this information from the raw data [25]. In general, these analyses rely upon reference datasets for spectra with known secondary structure, and it is necessary to benchmark a UV CD analysis method and reference set for a protein with a similar fold in order to obtain accurate results. Here, WT IsdG was used to benchmark the UV CD analysis because an X-ray crystal structure of this species is available (PDB ID $1 \mathrm{XBW}$ ) [32]. The X-ray crystal structure of WT IsdG was analyzed using the 
standard DSSP algorithm [33,34], resulting in an $\alpha$-helix content of $24 \%$ and a $\beta$-sheet content of $41 \%$ (Table S5). The UV CD spectrum of WT IsdG is consistent with the mixed $\alpha / \beta$ secondary structure since the minima at 225 and $210 \mathrm{~nm}$ associated with $\alpha$-helices can be observed as a trough and shoulder, respectively, superimposed on a broad negatively-signed feature associated with a $\beta$-sheet (Fig. 2). The SELCON3 algorithm with reference dataset 4 was previously used to successfully extract the secondary structure of MhuD [5, 2729], an enzyme with a similar fold to IsdG [35], so that approach was tested here. Based upon SELCON3 analysis of the UV CD spectrum, WT IsdG has 20 $\pm 7 \% \alpha$-helix and $41 \pm 7 \% \beta$-sheet content in aqueous solution. The differences between the X-ray crystallography and UV CD-derived secondary structure of WT IsdG are within the reported error for the algorithm and reference dataset. Thus, the combination of SELCON3 and reference dataset 4 is a reliable tool to extract secondary structure information from UV CD spectra of IsdG variants.

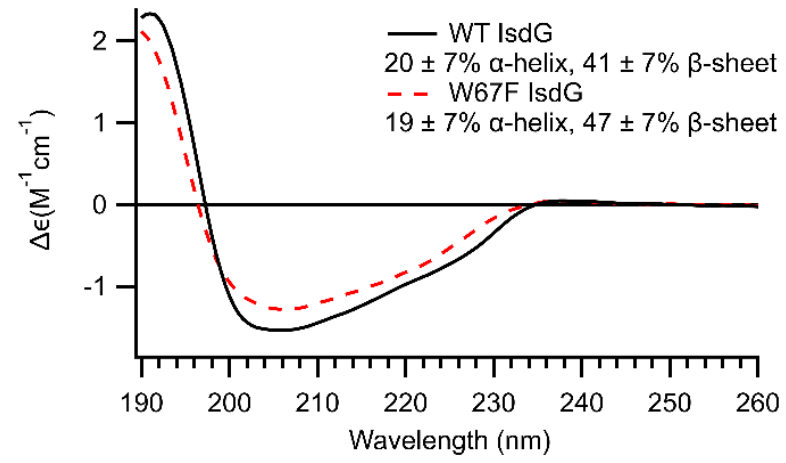

Fig. 2. The W67F substitution does not significantly alter the secondary structure of IsdG. UV CD spectra were acquired in $10 \mathrm{mM} \mathrm{KP}$ i $\mathrm{pH} 7.4$ and analyzed using the SELCON3 algorithm [7, 27-29].

Analysis of the UV CD data for W67F IsdG using this validated approach revealed that the amino acid substitution does not significantly alter the protein secondary structure. The UV CD spectrum of W67F IsdG is also consistent with a mixed $\alpha / \beta$ secondary structure (Fig. 2). SELCON3 analysis of these data yields an estimated secondary structure of $20 \pm 7 \% \alpha$-helix and $41 \pm 7 \% \beta$-sheet (Table S5) [27-29]. The predicted $1 \%$ decrease in $\alpha$-helical content and $6 \%$ increase in $\beta$-sheet content is within algorithmic error. Thus, the W67F substitution does not induce a significant secondary structural change. Furthermore, considering that IsdG is a $12.5 \mathrm{kDa}$ single-domain protein [ 30 , 32], the W67F substitution likely does not alter the protein tertiary structure either. Consequently, structural, electronic, and functional changes induced by the W67F substitution will be interpreted to arise from altered second-sphere interactions between IsdG and the heme substrate.

\subsection{The W67F substitution decreases heme ruffling induced by IsdG}

UV/Vis Abs spectroscopy was employed to identify the distal ligand to heme in W67F IsdG-heme. The UV/Vis Abs spectra of water- and hydroxideligated IsdG-heme have been reported in the literature. Water-bound IsdGheme is a high-spin species with an intense Soret band at $403 \mathrm{~nm}$, a moderately intense Q-band at $541 \mathrm{~nm}$, and a shoulder at $637 \mathrm{~nm} \mathrm{[10].} \mathrm{On} \mathrm{the} \mathrm{other} \mathrm{hand,}$ hydroxide-ligated IsdG-heme is a low-spin species with a Soret band at $412 \mathrm{~nm}$ and a vibronically-split Q band. At $\mathrm{pH} 7.4$, the UV/Vis Abs spectrum of W67F IsdG-heme exhibits a Soret band at $412 \mathrm{~nm}$ and a vibronically-split $\mathrm{Q}$ band (Fig. 3). This is consistent with a hydroxide-ligated heme substrate, which is also what is observed for WT enzyme [11]. Thus, the W67F substitution does not alter the protonation state of the distal ligand to iron.

$\mathrm{UV} / \mathrm{Vis}$ Abs spectroscopy was also employed to assess the influence of the W67F substitution on heme substrate ruffling by IsdG. In order to evaluate heme ruffling within the W67F IsdG active site, an established UV/Vis Abs- based assay was used to estimate heme ruffling changes arising from secondsphere substitutions for cyanide-bound heme [5]. An X-ray crystal structure for WT IsdG-heme-CN is not available, but heme ruffling in WT IsdG-heme-CN has been estimated to be $2.0 \AA$ based upon spectroscopically-validated $\mathrm{QM} / \mathrm{MM}$ calculations [7]. The UV/Vis Abs spectra of WT and W67F IsdGheme- $\mathrm{CN}$ are quite similar (Fig. 3), but closer inspection reveals that the W67F substitution blue-shifts both the Q and Soret bands by $100 \mathrm{~cm}^{-1}$. Based upon the QM/MM structure of WT IsdG-heme-CN, the UV/Vis Abs spectra of WT and W67F IsdG-heme-CN, and the established ruffling model noted above, the magnitude of heme ruffling in W67F IsdG-heme-CN was estimated to be 1.7 A. A similar decrease in heme ruffling was previously reported for IsdI-heme$\mathrm{CN}$ upon introduction of the W66Y substitution based upon X-ray crystallography (PDB IDs 3QGP and 4FNI) [1, 3], but it is important to note that IsdI induces more heme ruffling than IsdG. Altogether, these data reveal that the W67F substitution decreases heme ruffling by IsdG.

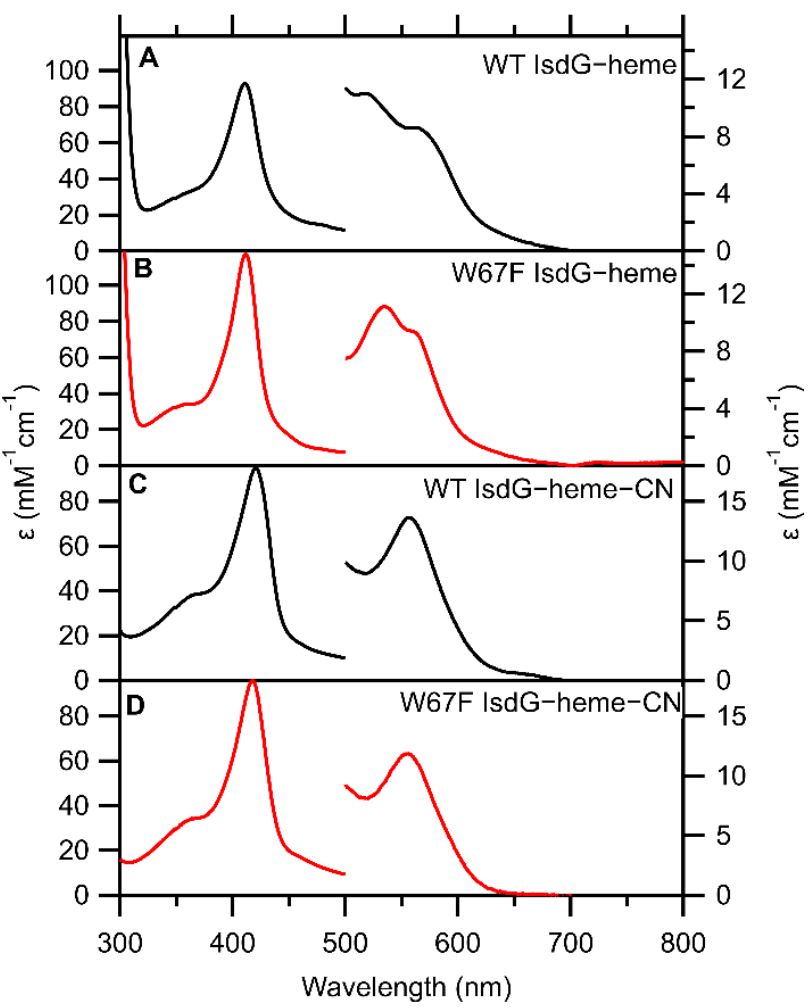

Fig. 3. UV/Vis Abs spectra of WT IsdG-heme (panel A), W67F IsdG-heme (panel B), WT IsdG-heme-CN (panel C), and W67F IsdG-heme-CN (panel D). IsdG-heme data was acquired in $50 \mathrm{mM} \mathrm{KP} \mathrm{i}_{\mathrm{i}} \mathrm{pH}$ 7.4, and IsdG-heme-CN data was acquired in $50 \mathrm{mM}$ Tris $\mathrm{pH} 7.4,150 \mathrm{mM} \mathrm{NaCl}$. At pH 7.4, both WT and W67F IsdG-heme are low-spin complexes with distal hydroxide ligands [10]. The W67F substitution induces a $100 \mathrm{~cm}^{-1}$ blue-shift of the heme Soret band, which can be attributed to decreased heme substrate ruffling [5].

\subsection{The W67F substitution perturbs the heme electronic structure}

The electronic structure of IsdG-heme-CN was investigated using ${ }^{1} \mathrm{H}$ NMR spectroscopy. ${ }^{1} \mathrm{H}$ NMR has been used successfully in the past to characterize the unusual electronic structures of IsdI-heme-CN and MhuDheme-CN $[1,4]$, and it was expected to provide similar insight into IsdGheme-CN. The ${ }^{1} \mathrm{H}$ NMR spectrum of WT IsdG-heme-CN contains several resonances outside the typical 0-10 ppm region (Fig. 4), which arise from hyperfine coupling between nuclear and electron spins [9]. Typically, several far downfield resonances arising from the heme methyl groups are observed in low-spin ferric hemes, but no ${ }^{1} \mathrm{H}$ resonances larger than $15 \mathrm{ppm}$ were observed for WT IsdG-heme-CN. Instead, several upfield hyperfine-shifted resonances 
are observed that have been assigned to the heme meso groups in low-spin, ferric heme species with ${ }^{2} B_{2 g}$ electronic ground states. Resonance assignments for IsdG-heme-CN were not made here, since the IsdG-heme-CN electronic structure will be further characterized using VTVH MCD below, but it is noteworthy that this ${ }^{1} \mathrm{H}$ NMR spectrum of IsdG-heme-CN is similar to that reported previously for IsdI-heme-CN. Based upon the similarities between the IsdG-heme- $\mathrm{CN}$ and IsdI-heme- $\mathrm{CN}{ }^{1} \mathrm{H}$ NMR spectra, the IsdG-heme-CN ground state is tentatively assigned to be ${ }^{2} B_{2 g}$, but further investigation using VTVH MCD is warranted.

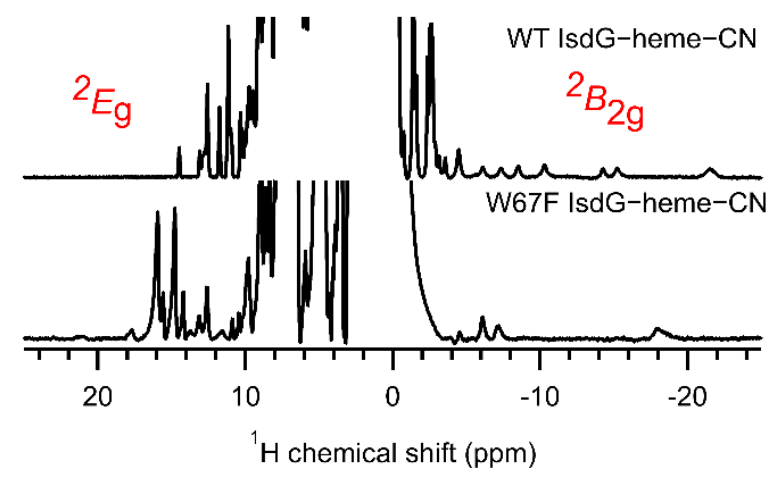

Fig. 4. ${ }^{1} \mathrm{H}$ NMR spectra of WT IsdG-heme-CN (top) and W67F IsdG-heme$\mathrm{CN}$ (bottom). ${ }^{1} \mathrm{H}$ NMR data was acquired in $10 \mathrm{mM} \mathrm{NaP}$ pH 7.4, $10 \% \mathrm{D}_{2} \mathrm{O}$ $(v / v)$ at $25^{\circ} \mathrm{C}$. Downfield hyperfine-shifted resonances for low-spin ferric heme have been associated with a ${ }^{2} E_{g}$ electronic state whereas upfield hyperfineshifted resonances have been associated with a ${ }^{2} B_{2 g}$ electronic state [9]. Thus, The W67F substitution increases the population of a ${ }^{2} E_{g}$ electronic state.

Next, the influence of the W67F substitution on the IsdG-heme-CN electronic structure was evaluated using ${ }^{1} \mathrm{H}$ NMR. Once again, the observation of hyperfine-shifted ${ }^{1} \mathrm{H}$ NMR resonances is clear evidence for a paramagnetic species (Fig. 4). Compared to WT enzyme, several additional downfield hyperfine-shifted resonances are observed in the ${ }^{1} \mathrm{H}$ NMR spectrum of W67F IsdG-heme-CN. The furthest downfield-shifted resonance is located at 17.8 ppm, which is similar to what was observed previously for W66Y IsdI-heme$\mathrm{CN}$ [3]. On the upfield side of the W67F IsdG-heme-CN ${ }^{1} \mathrm{H}$ NMR spectrum, the resonances observed at $-15.0,-10.4$, and $-8.5 \mathrm{ppm}$ for WT enzyme are no longer seen in the variant. Based upon a comparison of the WT IsdG-heme$\mathrm{CN}$ and IsdI-heme- $\mathrm{CN}{ }^{1} \mathrm{H}$ NMR spectra [1], these three upfield resonances likely arise from the heme meso groups of a low-spin, ferric heme in the ${ }^{2} B_{2 g}$ electronic state. The failure to observe these ${ }^{1} \mathrm{H}$ resonances in $\mathrm{W} 67 \mathrm{~F}$ IsdGheme- $\mathrm{CN}$ suggests that population of a ${ }^{2} B_{2 g}$ electronic state at room temperature is decreased in the W67F variant. However, line broadening arising from chemical exchange and paramagnetic relaxation can make ${ }^{1} \mathrm{H}$ NMR resonances difficult to detect, so MCD spectroscopy was employed to further investigate the electronic structure of IsdG-heme-CN.

\subsection{W67F IsdG-heme-CN has a ${ }^{2} B_{2 g}$ electronic ground state}

MCD spectroscopy was employed as a complementary approach to assess the influence of the W67F substitution on the electronic structure of IsdGheme-CN. The $5 \mathrm{~K}, 7$ T MCD spectrum of W67F IsdG-heme-CN was acquired and compared to published data for WT enzyme (Fig. 5) [10]. Several changes are apparent in the $13,000-21,000 \mathrm{~cm}^{-1}$ region, similar to those previously associated with an electronic state change from ${ }^{2} B_{2 g}$ to ${ }^{2} E_{g}$ upon introduction of the W66F substitution into MhuD-heme-CN [5]. In addition, a $100 \mathrm{~cm}^{-1}$ blueshift from 23,500 to $23,600 \mathrm{~cm}^{-1}$ of the intense, negatively-signed component of the Soret band was observed upon introduction of the W67F substitution. This spectral shift is consistent with what was observed using UV/Vis Abs spectroscopy (Fig. 3), and is further evidence that the heme substrate of W67F IsdG is less ruffled than the heme substrate of WT IsdG. Finally, the MCDdetected Soret band of W67F IsdG-heme-CN is four times more intense than that reported for WT enzyme. This intensity change has previously been attributed to increased population of a ${ }^{2} E_{g}$ state in the W66F variant of MhuDheme-CN. The additional MCD intensity arises from enhanced spin-orbit coupling between the two components of a ${ }^{2} E_{g}$ state[4]. Thus, both MCD and UV/Vis Abs data are consistent with a less ruffled heme, and MCD and ${ }^{1} \mathrm{H}$ NMR data are consistent with increased population of a ${ }^{2} E_{g}$ state, for W67F IsdG compared to WT enzyme.
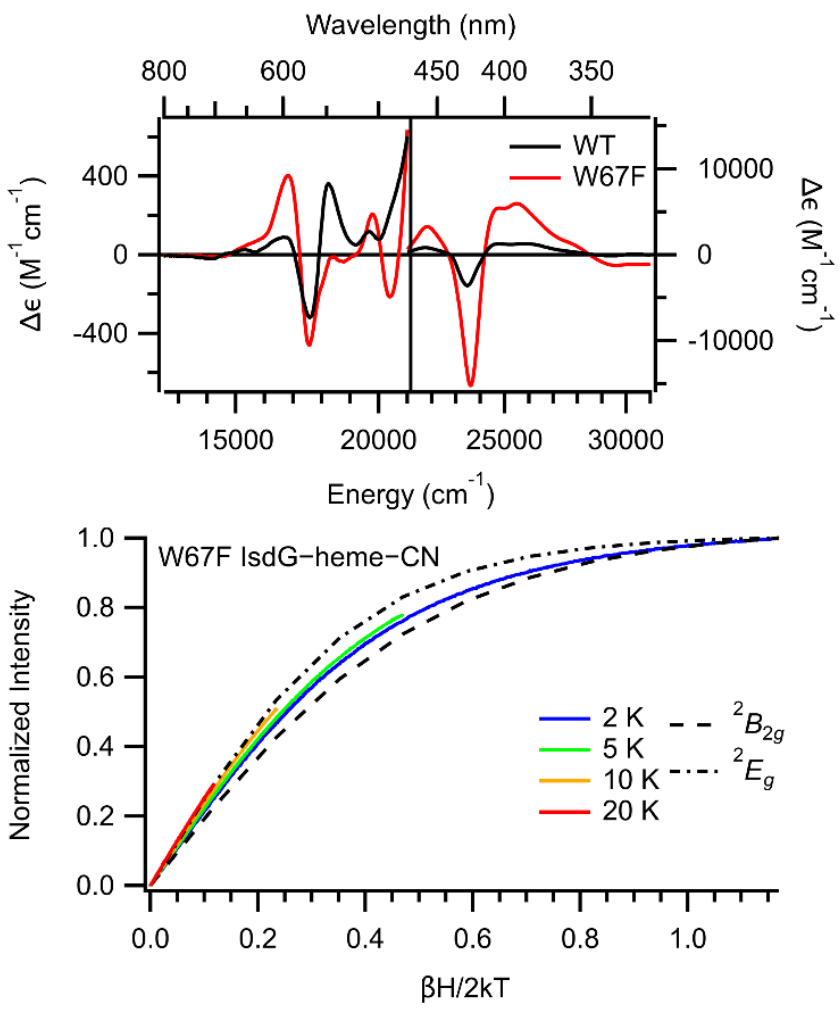

Fig. 5. $5 \mathrm{~K}, 7 \mathrm{~T}$ MCD spectra of WT and W67F IsdG-heme-CN (top), plus saturation magnetization curves for the $424 \mathrm{~nm}$ MCD band of W67F IsdGheme-CN (bottom) [10]. All data was acquired for samples is $50 \mathrm{mM} \mathrm{KP}$ i $\mathrm{pH}$ $7.4,150 \mathrm{mM} \mathrm{NaCl}, 60 \%$ glycerol $(v / v)$. The W67F substitution increases the population of a ${ }^{2} E_{g}$ electronic state, but retains a ${ }^{2} B_{2 g}$ electronic ground state.

The temperature and field dependence of the W67F IsdG-heme-CN MCD spectrum was analyzed to gain further insight into the electronic structure of this species. VTVH MCD has proven to be an exquisitely sensitive probe of transition metal electronic structure [36], and a framework has been established to readily interpret data for low-spin ferric heme [4]. It has been wellestablished since the advent of the so-called "Gouterman four-orbital model" that the heme Soret band is xy-polarized [37], which means that the magnetic field dependence of this band depends upon $g_{z}$. It has also been shown that different low-spin ferric heme electronic states have different $g_{z}$ values [9], and it is arguably easier to measure the values indirectly using VTVH MCD as compared to directly via EPR since VTVH MCD does not suffer from saturation effects that may make some EPR signals undetectable. The VTVH MCD curves for W67F IsdG-heme-CN are clearly consistent with an $S=1 / 2$ ground state based upon their similarities to simulated curves for two low-spin heme model complexes (Fig. 5) [38]. Notably, the 2, 5, and $10 \mathrm{~K}$ curves are "nested", which arises from thermal population of low-lying electronic excited states at cryogenic temperatures. The slope of the VTVH MCD saturation magnetization curves increases with increasing temperature, indicating that 
W67F IsdG-heme-CN has a ${ }^{2} B_{2 g}$ ground state and a ${ }^{2} E_{g}$ excited state. This is a surprising result since the signals arising from the ${ }^{2} E_{g}$ state dominate the ${ }^{1} \mathrm{H}$ NMR spectrum of W67F IsdG-heme-CN due to exchange broadening (Fig. 4), and illustrates the advantage of VTVH MCD as a heme electronic structure probe. Consequently, the electronic ground state of W67F IsdG-heme-CN is ${ }^{2} B_{2 g}$ and this species possesses a low energy ${ }^{2} E_{g}$ excited state that can be thermally populated.

\subsection{W66Y IsdI-heme-CN also has a ${ }^{2} B_{2 g}$ electronic ground state}

As the W66Y IsdI-heme-CN variant has a very similar ${ }^{1} \mathrm{H}$ NMR spectrum to that reported for W67F IsdG-heme-CN, the electronic structure of this species was investigated using MCD spectroscopy. X-ray crystal structures of both WT and W66Y IsdI-heme-CN (PDB IDs 3QGP and 4FNI) [1, 3]; this second-sphere substitution does not alter the secondary or tertiary structure of the enzyme. As noted above, the ${ }^{1} \mathrm{H}$ NMR spectrum of W66Y IsdI-heme-CN has downfield resonances previously assigned to the heme methyl groups of a ${ }^{2} E_{g}$ electronic state [3], but this study has shown that exchange broadening can mask signals arising from a ${ }^{2} B_{2 g}$ electronic state. There are several differences in the $13,000-21,000 \mathrm{~cm}^{-1}$ regions of the WT and W66Y IsdI-heme-CN MCD spectra (Fig. 6) [6], and these differences are very similar to those observed between WT and W67F IsdG-heme-CN (Fig. 5). Also similar to the observations for WT and W67F IsdG-heme- $\mathrm{CN}$, the Soret band MCD intensity increases four-fold from WT to W66Y IsdI-heme-CN. As noted above, these spectral changes can all be attributed to increased population of a ${ }^{2} E_{g}$ state in the variants. These data are consistent with a ${ }^{2} E_{g}$ ground state for W66Y IsdIheme- $\mathrm{CN}$, but they could also be explained by a subtler electronic structure change. If both WT and W66Y IsdI-heme-CN are thermal mixtures of ${ }^{2} E_{g}$ and ${ }^{2} B_{2 g}$ electronic states, then the ${ }^{1} \mathrm{H}$ NMR and MCD observations could be explained by a perturbation of the relative populations of the ${ }^{2} E_{g}$ and ${ }^{2} B_{2 g}$ electronic states. As ${ }^{1} \mathrm{H}$ NMR and MCD spectral data could not resolve the ambiguity regarding the electronic ground state of W66Y IsdI-heme- $\mathrm{CN}$, the temperature and field dependence of the MCD data were carefully examined.

VTVH MCD was employed to definitively assign the electronic ground state of W66Y IsdI-heme-CN. As was the case for W67F IsdG-heme-CN, the MCD saturation magnetization curves of W66Y IsdI-heme-CN are clearly consistent with an $S=1 / 2$ ground state (Fig. 6). Also similar to observations for W67F IsdG-heme-CN, the MCD saturation magnetization curves for W66Y IsdI-heme-CN are "nested" indicating thermal population of more than one $S$ $=1 / 2$ electronic state at cryogenic temperatures. Notably, the MCD saturation magnetization curves previously reported for WT IsdI-heme-CN are also "nested" [6], which reveals that both WT and W66Y IsdI-heme-CN are thermal mixtures of ${ }^{2} E_{g}$ and ${ }^{2} B_{2 g}$ electronic states. Finally, the slope of the MCD saturation magnetization curves for W66Y IsdI-heme- $\mathrm{CN}$ increases with increasing temperature, revealing that the electronic ground state for this species is ${ }^{2} B_{2 g}$. Thus, both WT and W66Y IsdI-heme-CN have ${ }^{2} B_{2 g}$ electronic ground states, and both WT and W66Y IsdI-heme-CN have thermallyaccessible ${ }^{2} E_{g}$ electronic excited states. However, the energy difference between the ${ }^{2} B_{2 g}$ and ${ }^{2} E_{g}$ states is smaller for W66Y IsdI-heme-CN resulting in greater population of the ${ }^{2} E_{g}$ state at physiologically-relevant temperatures.

\subsection{W67F IsdG has a diminished rate of heme oxygenation}

Next, established UV/Vis Abs assays were employed to ascertain the impact of the heme substrate geometric and electronic structure changes induced by the W67F substitution on IsdG activity. WT IsdG degrades heme upon the addition of ascorbate in the presence of catalase (Fig. 7) [30], to prevent non-enzymatic coupled oxidation [39]. After 60 minutes, the UV/Vis Abs spectrum of the reaction mixture is very similar to that reported previously for the IsdG product [15], which strongly suggests that WT IsdG degrades heme to staphylobilin under these conditions. When this assay was repeated for W67F
IsdG, a distinct Soret band at $412 \mathrm{~nm}$ was still observable after 60 minutes. This suggests that heme degradation by W67F is slower than for WT enzyme. In addition, a broad band centered at $680 \mathrm{~nm}$ gains intensity for the first 30 minutes following ascorbate addition and then decreases to approximately half its maximal intensity after an additional 30 minutes. We reasoned that this $680 \mathrm{~nm}$ band could be better resolved by repeating the assay with a higher enzyme concentration and a lower sodium ascorbate concentration. Indeed, for $30 \mu \mathrm{M}$ W67F IsdG and $1 \mathrm{mM}$ sodium ascorbate, a clear $680 \mathrm{~nm}$ band grows for the first 115 minutes of the assay before beginning to diminish. Absorbance at this wavelength is likely derived from production of an unstable biliverdin product [40], but further characterization is needed to reach a definitive conclusion. Nevertheless, based upon the UV/Vis Abs data presented here, it is clear that the W67F substitution alters the rate and/or mechanism of heme degradation by IsdG.
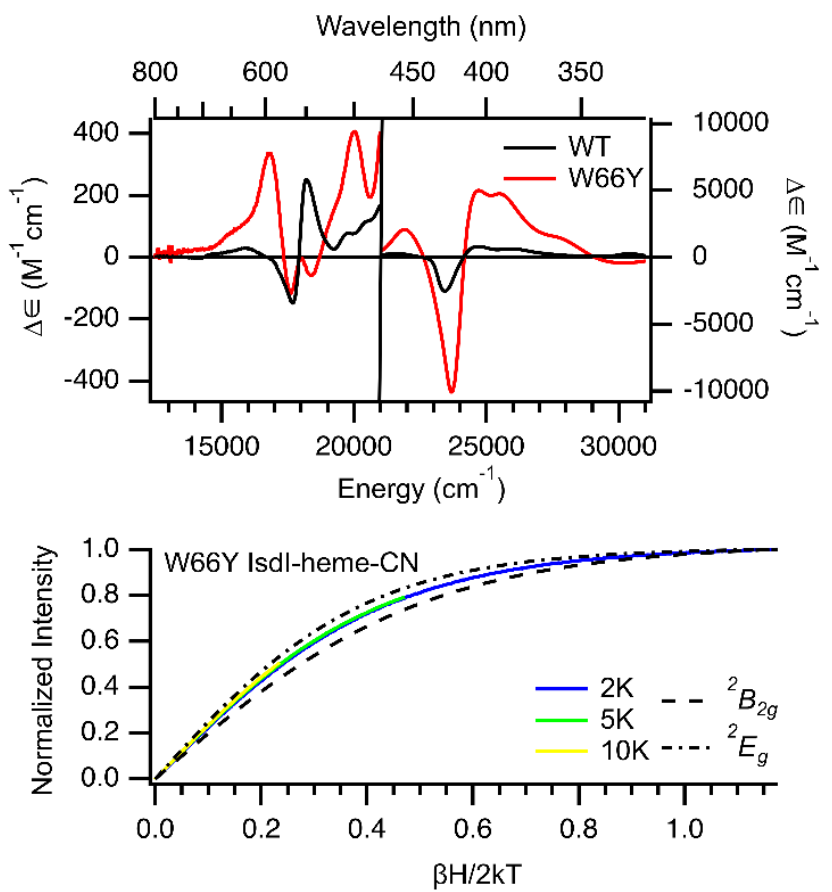

Fig. 6. $5 \mathrm{~K}, 7 \mathrm{~T}$ MCD spectra of WT and W66Y IsdI-heme-CN (top), plus saturation magnetization curves for the $422 \mathrm{~nm}$ MCD band of W66Y IsdIheme-CN (bottom) [6]. All data was acquired for samples is $50 \mathrm{mM} \mathrm{KP}$ i $\mathrm{pH}$ $7.4,150 \mathrm{mM} \mathrm{NaCl}, 60 \%$ glycerol $(v / v)$. The W66Y substitution increases the population of a ${ }^{2} E_{g}$ electronic state, but retains a ${ }^{2} B_{2 g}$ electronic ground state.

The pseudo-first order rates for heme oxygenation were extracted from the UV/Vis Abs assays. The Soret band arises from a $\pi \rightarrow \pi^{*}$ transition of the heme substrate [37], so the IsdG-catalyzed oxygenation of heme to mesohydroxyheme can be monitored by following the time-course of this band [30]. Kinetic analysis is complicated by the fact that IsdG intermediates and products absorb light at this wavelength [15], but these issues have been addressed in a recently reported analytical expression [20]. This analytical expression corrects for non-zero absorbance at infinite time, which is especially important when comparing variants that yield spectroscopically distinct products. Based upon the data reported here (Fig. 7), and the kinetic model reported previously, WT IsdG oxygenates heme with a pseudo-first order rate constant of $0.111 \pm 0.004$ $\mathrm{min}^{-1}$. Upon introduction of the W67F substitution, the rate decreases to 0.049 $\pm 0.006 \mathrm{~min}^{-1}$. Thus, IsdG-catalyzed heme oxygenation shows the same substrate ruffling-dependence that was previously reported for two other noncanonical heme oxygenases: IsdI and MhuD [3, 5]. Furthermore, heme 
oxygenation by IsdG is markedly faster than oxygenation by MhuD, which may be derived from the observation that the IsdG active site induces more heme substrate ruffling than the MhuD active site. Clearly, the W67F substitution decreases the rate of heme oxygenation by IsdG and the likely origin of this observation is reduced heme ruffling.
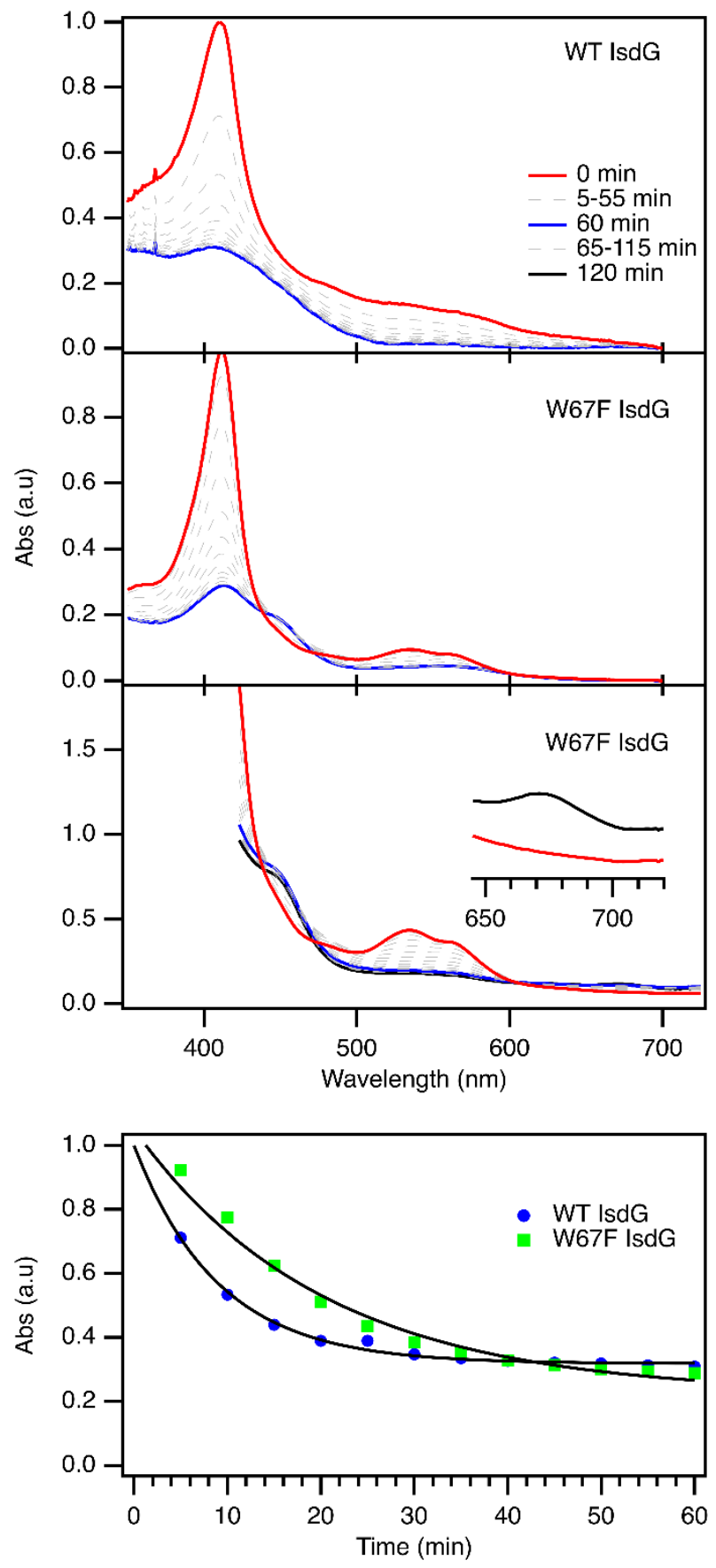

Fig. 7. Ascorbate-mediated, aerobic degradation of heme by WT IsdG and W67F IsdG (top), and fits of the time-course for the Soret band to a pseudofirst order kinetic model (bottom). IsdG-catalyzed heme degradation by $10 \mu \mathrm{M}$ enzyme was monitored for $60 \mathrm{~min}$ in $50 \mathrm{mM} \mathrm{KP}$ pH 7.4. In addition, heme degradation by $30 \mu \mathrm{M}$ W67F IsdG was monitored for $120 \mathrm{~min}$. The rate of heme degradation by $10 \mu \mathrm{M}$ WT enzyme $\left(0.111 \pm 0.004 \mathrm{~min}^{-1}\right)$ is more than double that of the $10 \mu \mathrm{M}$ W67F variant $\left(0.049 \pm 0.006 \mathrm{~min}^{-1}\right)$.

\subsection{W67F IsdG converts heme to biliverdin}

ESI-MS was employed to identify the product(s) of IsdG-catalyzed heme degradation. Previous work has identified staphylobilins as the major product of IsdG based upon product isolation and characterization [13]. However, a recent in proteo MS study of the non-canonical HO MhuD revealed that the product isolation approach can fail to detect alternate enzyme products [20]. Indeed, ESI-MS of the WT IsdG reaction mixture $60 \mathrm{~min}$ after the addition of ascorbate reveals a mixture of $\mathrm{m} / \mathrm{z} 583,599$, and 611 species (Fig. 8). The 599 and $611 \mathrm{Da}$ species were previously observed for IsdG-catalyzed heme degradation [15], and attributed to the staphylobilin product and formyloxobilin intermediate, respectively. However, the 583 Da species was not reported previously. This molecular weight corresponds to biliverdin, which is the major product of canonical HOs and minor product of MhuD [19, 41]. The in proteo MS data is not rigorously quantitative, but biliverdin appears to represent $\sim 10 \%$ of the IsdG product distribution. This level of biliverdin production by IsdG is consistent with the previous observation that $\mathrm{CO}$ represents $14 \pm 2 \%$ of the $\mathrm{C} 1$ products for this enzyme [14]. Thus, IsdG degrades heme to a mixture of staphylobilin and biliverdin products.

Finally, the products of W67F IsdG-catalyzed heme degradation were analyzed with ESI-MS. After $60 \mathrm{~min}$, the W67F IsdG reaction intermediate is dominated by a mixture of $\mathrm{m} / \mathrm{z} 583,611$, and 616 species (Fig. 8). These ions correspond to biliverdin [19], formyloxobilin [15], and heme, respectively. The observation of a significant fraction of unreacted heme substrate is consistent with the decreased oxygenation rate determined above (Fig. 7). More surprisingly, a major component of the W67F IsdG reaction mixture is formyloxobilin, but no staphylobilin is observed. These data suggest that the W67F substitution also either alters the distribution of formyloxobilin isomers or inhibits the conversion of formyloxobilin to staphylobilin. Finally, it is particularly noteworthy that biliverdin product by W67F IsdG is increased compared to WT enzyme. This result is consistent with the observation of a broad band centered at $680 \mathrm{~nm}$ in the UV/Vis Abs-monitored assay. Based on these data, it is clear that the W67F substitution alters the IsdG mechanism resulting in a novel product distribution.

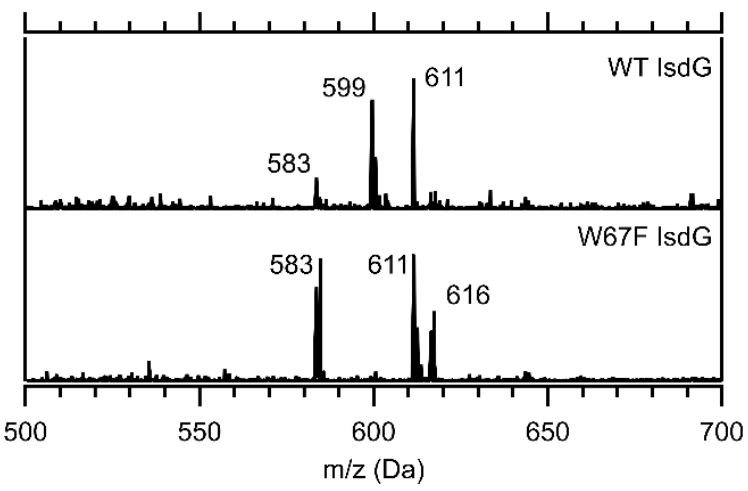

Fig. 8. Ascorbate-mediated, aerobic degradation of heme by WT IsdG (top) and W67F IsdG (bottom). The product distributions were assessed using ESIMS after $60 \mathrm{~min}$ of reaction time in $50 \mathrm{mM} \mathrm{KP}$ i $\mathrm{pH}$ 7.4. Prior to ESI-MS, samples were eluted from a $\mathrm{C}_{18}$ column with a $\mathrm{MeCN}$ gradient. Data were acquired for $\mathrm{m} / \mathrm{z} 600-1500$, but only the $\mathrm{m} / \mathrm{z} 500-700$ region is shown here for clarity. The major products of WT IsdG are staphylobilin (599 Da) and formyloxobilin $(611 \mathrm{Da})$. On the other hand, the major products of W67F IsdG are biliverdin $(583 \mathrm{Da})$ and formyloxobilin $(611 \mathrm{Da})$. 


\section{Discussion}

\subsection{Heme substrate structure in non-canonical heme oxygenases}

A relatively complete picture for the structural role of the conserved second-sphere Trp of non-canonical heme oxygenases is now available. The $\mathrm{X}$ ray crystal structures of IsdI-heme-CN and MhuD-heme-CN (PDB IDs 3QGP and 4NL5) revealed that these enzymes induce a $b_{1 u}$ out-of-plane ruffling distortion of their heme substrates from planarity $[1,4]$. More recently, a spectroscopically-validated model of IsdG-heme-CN showed that the IsdG active site generates an intermediate amount of heme ruffling $(2.0 \AA)$ compared to IsdI $(2.3 \AA)$ and MhuD (1.4 $\AA$, Fig. 9) [3, 7]. Previous studies have shown that substituting a Tyr or Phe for the conserved second-sphere Trp residue decreases the average amount of heme substrate ruffling by $0.4-0.8 \AA$ in IsdI and MhuD [5], and the data presented here shows that this same substitution decreases heme ruffling by $0.3 \AA$ in IsdG (Fig. 3). Thus, the structural role of the second-sphere Trp is conserved between IsdG, IsdI, and MhuD; steric interactions between a second-sphere Trp and the heme substrate induce a $b_{1 u}$ out-of-plane ruffling deformation of heme.

However, simply stating that the second-sphere Trp induces a ruffling deformation of heme is an over-simplification of the underlying structural dynamics. MCD experiments have demonstrated that the heme substrate ruffling distortions measured using X-ray crystallography represent a weighted average of two distinct substrate conformations [6]. To be specific, cyanideand histidine-ligated ferric heme has "planar" (0.5 Å ruffling distortion) and "ruffled" (1.6 ̊̊ ruffling distortion) conformations separated by an energy barrier of $1.2 \mathrm{kcal} / \mathrm{mol}$. Previously reported ${ }^{1} \mathrm{H}$ NMR, MCD, and multireference perturbation theory data have shown that the W66F substitution decreases the average ruffling of heme in MhuD by altering the relative populations of the "planar" and "ruffled" conformations [4, 5]. Here, new MCD data for IsdG and IsdI reveal that the average heme ruffling changes in these enzymes also arise from altered conformer populations (Figs. 5 and 6). Consequently, the most accurate description of heme within non-canonical heme oxygenase active sites is that of a dynamic substrate that rapidly samples both "planar" and "ruffled" conformations. The second-sphere interactions between the conserved Trp residue and the heme modulate the ratio of "planar" and "ruffled" substrate.

It is worth noting that the orientation of the heme substrate relative to the conserved second-sphere Trp residue is different in IsdG, IsdI, and MhuD. In the X-ray crystal structure of WT IsdI-heme-CN (PDB ID 3QGP), a single heme orientation was observed with a steric contact between the $\beta$-meso carbon of heme and the Trp66 side-chain [1]. In contrast, the heme substrate is rotated by $\sim 90^{\circ}$ in WT MhuD-heme-CN (PDB ID 4NL5), resulting in a steric contact between the $\alpha$-meso carbon of heme and the Trp66 side-chain [4]. The exact orientation of heme in the X-ray crystal structures of N7A IsdG-heme (PDB ID 2ZDO) and W66Y IsdI-heme-CN (PDB ID 4FNI) is unclear due to disorder in the electron density maps noted by the crystallographers [3, 42], but the secondsphere Trp residue is certainly in contact with either the $\beta$ - or $\delta$-meso carbons of heme in those species. Regardless, any of these contacts induce the same ruffling deformation of the heme substrate since the $b_{1 u}$ out-of-plane normal mode responsible for heme ruffling induces coupled distortion of all four heme meso carbons [2]. The $\sim 90^{\circ}$ rotation of heme in MhuD compared to IsdG and IsdI would certainly be expected to alter the regiochemistry of heme hydroxylation. But, all three enzymes catalyze hydroxylation of the $\beta$ - and $\delta$ meso carbons $[13,18]$. It appears that an altered structural role for the secondsphere Asn residue offsets the rotation of heme within the enzyme active site. In IsdG Asn7 forms a hydrogen bond with the iron-ligating $\alpha$-oxygen of ferricperoxoheme [10], whereas in MhuD Asn7 has been proposed to form a hydrogen bond with the terminal $\beta$-oxygen of ferric-peroxoheme based upon QM/MM calculations [21]. Thus, the altered orientations of heme within the non-canonical heme oxygenase active sites, and the altered hydrogen bonding interactions with Asn7, may combine to orient the distal peroxo ligand along the $\beta / \delta$-meso carbon axis of heme in all three enzymes. Nevertheless, further experimental characterization of the structural role for Asn7 within the MhuD active site is warranted before this conclusion can be reached definitively.

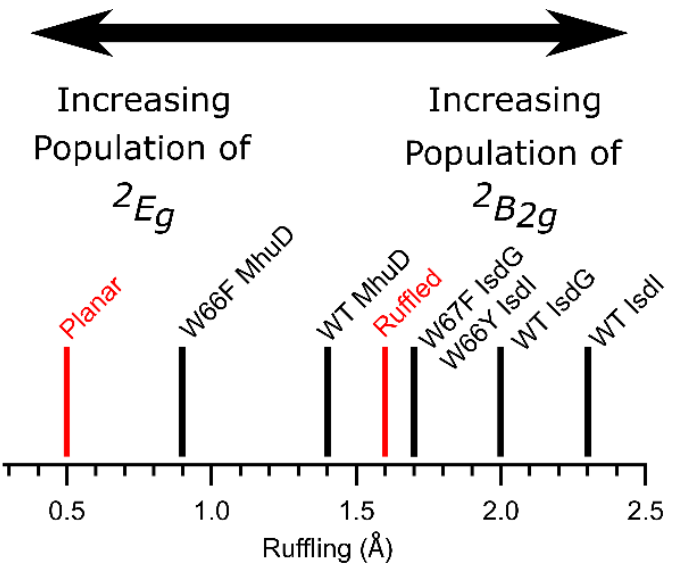

Fig. 9. Cyanide-inhibited, non-canonical heme oxygenases with average heme ruffling distortions ranging from 0.7 to $2.3 \AA$ have been prepared [1, 3-5, 7]. According to multi-reference perturbation theory calculations: the "planar" conformation of cyanide- and histidine-ligated ferric heme exhibits $0.5 \AA$ of ruffling, the "ruffled" conformation exhibits $1.6 \AA$ of ruffling, and the transition state between the two conformations has $1.0 \AA$ of ruffling [6]. The electronic ground state of all six variants of cyanide-inhibited, non-canonical heme oxygenases are accurately predicted by the theoretical model, and all six variants stabilize a thermal mixture of the two heme conformations.

\subsection{Unusual heme electronic structure in non-canonical heme oxygenases}

A relatively complete picture of how the conserved second-sphere Trp of non-canonical heme oxygenases tunes the electronic structure of the heme substrate is also now available. Several years ago MCD characterization of MhuD-heme-CN revealed that this species is a thermal mixture of two electronic states: a ${ }^{2} B_{2 g}$ ground state with a $\left(3 d_{\mathrm{xz}, \mathrm{yz}}\right)^{4}\left(3 d_{\mathrm{xy}}\right)^{1}$ Fe electron configuration, and a low-lying ${ }^{2} E_{g}$ excited state with a $\left(3 d_{\mathrm{xy}}\right)^{2}\left(3 d_{\mathrm{xz}, \mathrm{yz}}\right)^{3} \mathrm{Fe}$ electron configuration (Fig. 9) [4]. Subsequent studies revealed that the ${ }^{2} B_{2 g}$ and ${ }^{2} E_{g}$ electronic states are associated with the "ruffled" and "planar" heme conformations, respectively [6], and the same thermal mixture of a ${ }^{2} B_{2 g}$ ground state and a ${ }^{2} E_{g}$ excited state is present in IsdG and IsdI [10]. It was previously shown that the W66F substitution swaps the relative energies of the "planar" and "ruffled" heme conformations resulting in a ${ }^{2} E_{g}$ ground state with a thermally-accessible ${ }^{2} B_{2 g}$ excited state for W66F MhuD-heme-CN [5]. The data presented here reveals that the influence of a second-sphere Trp substitution is more subtle for IsdG and IsdI: the W67F and W66Y variants of IsdG and IsdI, respectively, retain a ${ }^{2} B_{2 g}$ ground state but have more populated ${ }^{2} E_{g}$ excited states owing to a decreased energy difference between the two conformations. Thus, although the exact details vary from enzyme to enzyme, the ruffling deformation induced by the conserved second-sphere Trp of noncanonical heme oxygenases generally increases the population of a ${ }^{2} B_{2 g}$ electronic state.

The electronic structure changes observed for cyanide-inhibited noncanonical heme oxygenases can be readily understood in terms of group theory. A $b_{1 u}$ ruffling distortion breaks $D_{4 h}$ symmetry and allows mixing of the Fe $3 d_{\mathrm{xy}}$ and porphyrin $a_{2 u}$ orbitals [37]. This mixing results in an electronic ground state change is observed when this ruffling-induced orbital mixing is large enough to raise the energy of the $\mathrm{Fe} 3 d_{\mathrm{xy}}$ orbital above the $\mathrm{Fe} 3 d_{\mathrm{xz}}$ and $3 d_{\mathrm{yz}}$ orbitals [9]. The ground state change is aided by the $\pi^{*}$-acceptor character of cyanide, since an axial $\pi^{*}$-acceptor will lower the energies of the Fe $3 d_{\mathrm{xz}}$ and $3 d_{\mathrm{yz}}$ orbitals. 
One might question the functional relevance of electronic structure changes for cyanide-inhibited enzyme when the ferric-peroxoheme species is believed to be the catalytically-relevant intermediate $[16,18]$. However, although peroxide and azide are $\pi$ donor ligands, which will raise the energies of the $\mathrm{Fe} 3 d_{\mathrm{xz}}$ and $3 d_{y z}$ orbitals and inhibit an electronic ground state change, ruffling will still induce mixing of the Fe $3 d_{x y}$ and porphyrin $a_{2 u}$ orbitals in these cases [7]. This electronic structure perturbation is unlikely to yield the wholesale ground state changes observed for cyanide-inhibited species, but it will still result in partial porphyrin-to-iron electron transfer. Consequently, the heme electronic structure change introduced in the "ruffled" substrate conformation of non-canonical heme oxygenases will favor hydroxylation of the meso carbons for the catalytically-relevant ferric-peroxoheme intermediate.

\subsection{Functional role of a conserved second-sphere Trp residue}

A general correlation between heme substrate conformation and heme oxygenation rate has been identified for non-canonical heme oxygenases. IsdG, IsdI, and MhuD have all been shown to convert heme to $\beta$ - and $\delta$-mesohydroxyheme $[13,15,18]$ It was previously shown that the rate of IsdIcatalyzed heme degradation depends upon the average amount of heme ruffling induced by a particular variant [3], and this work clarifies that the rate depends upon population of the "ruffled" substrate conformation (Fig. 6). It was also previously shown that the rate of MhuD-catalyzed heme monooxygenation depends upon the population of the "ruffled" substrate conformation [20], although this conclusion relied upon the development of a new analytical expression to analyze kinetic data acquired for several MhuD variants. Here, this new analytical expression was used to analyze kinetic data for IsdG variants revealing that the rate of IsdG-catalyzed heme oxygenation also depends upon the population of a "ruffled" substrate conformation (Fig. 7) and, furthermore, the correlation between heme oxygenation rate and the population of the "ruffled" conformation extends to a comparison between WT IsdG and MhuD. Although it remains unclear which step(s) of IsdG-catalyzed heme oxygenation are ruffling-dependent, these data can be understood in terms of a concerted rearrangement mechanism previously proposed for IsdG-catalyzed heme monooxygenation (Fig. 10) [10, 16], with the additional caveat that this mechanism is only accessible to the "ruffled" substrate conformation. There is not a general steric explanation for the correlation between ruffling and monooxygenation rate for non-canonical heme oxygenases because the distance between the terminal oxygen of ferric-peroxoheme and the $\beta / \delta$-meso carbons of porphyrin is longer for the "ruffled" conformation of MhuD [4]. Instead, the most general explanation is electronic: ruffling favors mixing of the $\mathrm{Fe} 3 d_{\mathrm{xy}}$ and porphyrin $a_{2 u}$ orbitals [6], which triggers partial porphyrin to iron charge transfer [5], which increases the electrophilicity of the heme meso carbons [12].

It is also now possible to identify a clear correlation between population of the "ruffled" substrate conformation and product identity for non-canonical heme oxygenases. It was previously shown that the major products of WT IsdG and WT MhuD are staphylobilin and mycobilin, respectively [13, 17]. Recently, it was realized that a second-sphere variant of $\mathrm{MhuD}$ that decreases population of the "ruffled" conformation, W66F, changes the major product of MhuD from mycobilin to biliverdin [20]. The ESI-MS data reported here reveals that decreased population of the "ruffled" conformation of IsdG induced by the W67F substitution also increases production of biliverdin (Fig. 8). These changes to product distributions could potentially be attributed to altered regioselectivity for the initial heme monooxygenation by invoking a stepwise radical mechanism (Fig. 10) [43], but additional data for enzymatic reactions initiated from regiochemically-defined intermediates would be needed to parse this level of mechanistic detail [18]. It is also worth noting that the W67F substitution fully suppresses the conversion of formyloxobilin to staphylobilin by IsdG. Staphylobilin is formed by rapid deformylation of formyloxobilin [15], and this reaction appears to require access to a "ruffled" substrate conformation. Many mechanistic questions remain to be answered for non- canonical heme oxygenases, but it is now clear that the conversions of heme to staphylobilin and mycobilin by these enzymes depend upon ruffling of the substrate.

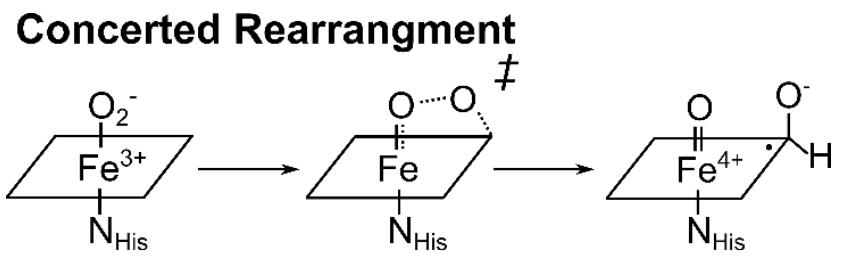

\section{Stepwise Radical}

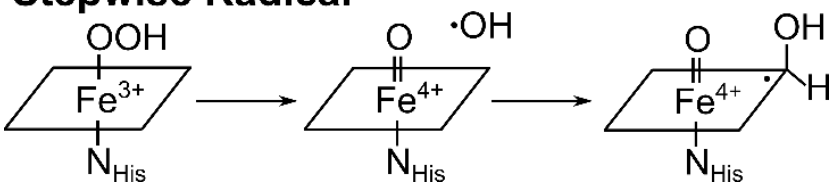

Fig. 10. Two possible mechanisms for heme monooxygenation by noncanonical heme oxygenases. The concerted rearrangement mechanism is the most consistent with available data for WT IsdG, IsdI, and MhuD. An alternative, stepwise radical mechanism could explain the altered product distributions for W66F MhuD and W67F IsdG.

\section{Conclusion}

It is now possible to make several general statements regarding the role of the conserved second-sphere Trp of non-canonical heme oxygenases. First, the steric interaction between Trp and heme favors population of a "ruffled" substrate conformation over the more typical "planar" substrate conformation. Second, these two substrate conformations are in dynamic equilibrium and the geometric changes are coupled with electronic changes, namely, the "ruffled" conformation promotes partial porphyrin-to-iron electron transfer. Finally, enzymatic rates and product distributions depend upon the relative populations of the "planar" and "ruffled" substrate conformations.

\section{Declaration of Competing Interest}

The authors declare that they have no known competing financial interests or personal relationships that could have appeared to influence the work reported in this paper.

\section{Acknowledgement}

This work is supported by the National Institutes of Health, National Institute of General Medical Sciences [Award R35-GM139516]. A.R.C. thanks the University of Vermont for financial support. The authors thank Bruce O'Rourke (University of Vermont) for assistance with ESI-MS data acquisition. In addition, the authors thank the Vermont Cancer Center DNA analysis facility for sequencing the IsdG and IsdI variants generated by this work

\section{Appendix A. Supplementary data}

Supplementary data to this article can be found online.

\section{References}

[1] S.J. Takayama, G. Ukpabi, M.E. Murphy, A.G. Mauk, Electronic properties of the highly ruffled heme bound to the heme degrading enzyme IsdI, Proc. $\begin{array}{llllll}\text { Natl. } & \text { Acad. } & \text { Sci. } & 108 & \text { (2011) } & \text { 13071-13076, }\end{array}$ https://doi.org/10.1073/pnas.1101459108. 
[2] W. Jentzen, X.-Z. Song, J.A. Shelnutt, Structural characterization of synthetic and protein-bound porphyrins in terms of the lowest-frequency normal coordinates of the macrocycle, J. Phys. Chem. B 101 (1997) 1684-1699, https://doi.org/10.1021/ip963142h.

[3] G. Ukpabi, S.J. Takayama, A.G. Mauk, M.E. Murphy, Inactivation of the heme degrading enzyme IsdI by an active site substitution that diminishes heme $\begin{array}{lllll}\text { ruffling, J. } & \text { Biol. } & \text { Chem. } 287 & \text { (2012) 34179-34188, }\end{array}$ https://doi.org/10.1074/jbc.M112.393249.

[4] A.B. Graves, R.P. Morse, A. Chao, A. Iniguez, C.W. Goulding, M.D. Liptak, Crystallographic and spectroscopic insights into heme degradation by Mycobacterium tuberculosis MhuD, Inorg. Chem. 53 (2014) 5931-5940, https://doi.org/10.1021/ic500033b.

[5] A.B. Graves, M.T. Graves, M.D. Liptak, Measurement of heme ruffling changes in MhuD using UV/Vis spectroscopy, J. Phys. Chem. B 120 (2016) 3844-3853, https://doi.org/10.1021/acs.jpcb.6b01497.

[6] A.B. Graves, E.H. Horak, M.D. Liptak, Dynamic ruffling distortion of the heme substrate in non-canonical heme oxygenase enzymes, Dalton Trans. (2016) 10058-10067, https://doi.org/10.1039/C6DT00702C.

[7] M.A. Conger, A.R. Cornetta, M.D. Liptak, Spectroscopic evidence for electronic control of heme hydroxylation by IsdG, Inorg. Chem. 58 (2019) 15455-15465, https://doi.org/10.1021/acs.inorgchem.9b02530.

[8] M. Nakamura, Electronic structures of highly deformed iron(III) prophyrin complexes, Coord. Chem. Rev. 250 (2006) 2271-2294, https://doi.org/10.1016/i.ccr.2006.03.001.

[9] F.A. Walker, Magnetic spectroscopic (EPR, ESEEM, Mössbauer, MCD and NMR) studies of low-spin ferriheme centers and their corresponding heme proteins, Coord. Chem. Rev. 185-186 (1999) 471-534, https://doi.org/10.1016/S0010-8545(99)00029-6.

[10] C.L. Lockhart, M.A. Conger, D. Pittman, M.D. Liptak, Hydrogen bond donation to the heme distal ligand of Staphylococcus aureus IsdG tunes the electronic structure, J. Biol. Inorg. Chem. 20 (2015) 757-770, https://doi.org/10.1007/s00775-015-1263-5.

[11] S. Takahashi, S. Nambu, T. Matsui, H. Fujii, H. Ishikawa, Y. Mizutani, K. Tsumoto, M. Ikeda-Saito, Unique electronic structures of the highly ruffled hemes in heme-degrading enzymes of Staphylococcus aureus, IsdG and IsdI, by resonance Raman and electron paramagnetic resonance spectroscopies, $\begin{array}{llll}\text { Biochemistry } & 59 & \text { (2020) 3918-3928, }\end{array}$ https://doi.org/10.1021/acs.biochem.0c00731.

[12] M. Rivera, G.A. Caignan, A.V. Astashkin, A.M. Raitsimring, T.K. Shokhireva, F.A. Walker, Models of the low-spin iron(III) hydroperoxide intermediate of heme oxygenase: magnetic resonance evidence for thermodynamic stabilization of the $\mathrm{d}_{x y}$ electronic state at ambient temperatures, J. Am. Chem. Soc. 124 (2002) 6077-6089, https://doi.org/10.1021/ja017334o. [13] M.L. Reniere, G.N. Ukpabi, S.R. Harry, D.F. Stec, R. Krull, D.W. Wright, B.O. Bachmann, M.E. Murphy, E.P. Skaar, The IsdG-family of haem oxygenases degrades haem to a novel chromophore, Mol. Microbiol. 75 (2010) 1529-1538, https://doi.org/10.1111/j.1365-2958.2010.07076.X.

[14] T. Matsui, S. Nambu, Y. Ono, C.W. Goulding, K. Tsumoto, M. IkedaSaito, Heme degradation by Staphylococcus aureus IsdG and IsdI liberates formaldehyde rather than carbon monoxide, Biochemistry 52 (2013) 30253027, https://doi.org/10.1021/bi400382p.

[15] B.R. Streit, R. Kant, M. Tokmina-Lukaszewska, A.I. Celis, M.M Machovina, E.P. Skaar, B. Bothner, J.L. DuBois, Time-resolved studies of IsdG protein identify molecular signposts along the non-canonical heme oxygenase $\begin{array}{lllll}\text { pathway, J. } & \text { Biol. } & \text { Chem. } & & \end{array}$ https://doi.org/10.1074/jbc.M115.666560.

[16] S.J. Takayama, S.A. Loutet, A.G. Mauk, M.E. Murphy, A ferric-peroxo intermediate in the oxidation of heme by IsdI, Biochemistry 54 (2015) 2613 2621, https://doi.org/10.1021/acs.biochem.5b00239.

[17] S. Nambu, T. Matsui, C.W. Goulding, S. Takahashi, M. Ikeda-Saito, A new way to degrade heme: the Mycobacterium tuberculosis enzyme MhuD catalyzes heme degradation without generating CO, J. Biol. Chem. 288 (2013) 10101-10109, https://doi.org/10.1074/ibc.M112.448399.

[18] T. Matsui, S. Nambu, C.W. Goulding, S. Takahashi, H. Fujii, M. IkedaSaito, Unique coupling of mono- and dioxygenase chemistries in a single active site promotes heme degradation, Proc. Natl. Acad. Sci. 113 (2016) 3779-3784, https://doi.org/10.1073/pnas.1523333113.

[19] A. Chao, C.W. Goulding, A single mutation in the Mycobacterium tuberculosis heme-degrading protein, MhuD, results in different products, $\begin{array}{llll}\text { Biochemistry } & 58 & \text { (2019) 489-492, }\end{array}$ https://doi.org/10.1021/acs.biochem.8b01198.

[20] B. Thakuri, B.D. O'Rourke, A.B. Graves, M.D. Liptak, A dynamic substrate is required for MhuD-catalyzed degradation of heme to mycobilin,
Biochemistry

60

(2021)

918-928,

https://doi.org/10.1021/acs.biochem.0c00892.

[21] C. Yuan, Y. Zhang, H. Tan, X. Li, G. Chen, Z. Jia, ONIOM investigations of the heme degradation mechanism by MhuD: the critical function of heme ruffling, Phys. Chem. Chem. Phys. 22 (2020) 8817-8826, https://doi.org/10.1039/C9CP05868K.

[22] M.A. Conger, D. Pokhrel, M.D. Liptak, Tight binding of heme to Staphylococcus aureus IsdG and IsdI precludes design of a competitive inhibitor, Metallomics 9 (2017) 556-563, https://doi.org/10.1039/c7mt00035a. [23] R.B. Kapust, J. Tözsér, J.D. Fox, D.E. Anderson, S. Cherry, T.D. Copeland, D.S. Waugh, Tobacco etch virus protease: mechanism of autolysis and rational design of stable mutants with wild-type catalytic proficiency, Protein Eng. 14 (2001) 993-1000, https://doi.org/10.1093/protein/14.12.993. [24] S. Nallamsetty, R.B. Kapust, J. Tözsér, S. Cherry, J.E. Tropea, T.D. Copeland, D.S. Waugh, Efficient site-specific processing of fusion proteins by tobacco vein mottling virus protease in vivo and in vitro, Protein Expression Purif. 37 (2004) 108-115, https://doi.org/10.1016/j.pep.2004.08.016.

[25] L. Whitmore, B.A. Wallace, Protein secondary structure analyses from circular dichroism spectroscopy: methods and reference databases, Biopolymers 89 (2008) 392-400,

[26] L. Whitmore, B.A. Wallace, DICHROWEB: an online server for protein secondary structure anlyses from circular dichroism spectroscopic data, Nucleic Acids Res. 32 (2004) W668-W673, https://doi.org/10.1002/bip.20853.

[27] N. Sreerama, R.W. Woody, A self-consistent method for the analysis of protein secondary structure from circular dichroism, Anal. Biochem. 209 (1993) 32-44, https://doi.org/10.1006/abio.1993.1079.

[28] N. Sreerama, S.Y. Venyaminov, R.W. Woody, Estimation of the Number of $\alpha$-helical and $\beta$-strand Segments in Proteins using Circular Dichroism $\begin{array}{lllll}\text { Spectroscopy, } & \text { Protein } & \text { Science } & 8 & \text { (1999) 370-380, }\end{array}$ https://doi.org/10.1110/ps.8.2.370.

[29] N. Sreerama, R.W. Woody, Estimation of protein secondary structure from circular dichroism spectra: comparison of CONTIN, SELCON and CDSSTR methods with an expanded reference set, Anal. Biochem. 287 (2000) 252-260, https://doi.org/10.1006/abio.2000.4880.

[30] E.P. Skaar, A.H. Gaspar, O. Schneewind, IsdG and IsdI, heme-degrading enzymes in the cytoplasm of Staphylococcus aureus, J. Biol. Chem. 279 (2004) 436-443, https://doi.org/10.1074/jbc.M307952200.

[31] E.A. Berry, B.L. Trumpower, Simultaneous Determination of Hemes $a, b$, and $c$ from Pyridine Hemochrome Spectra, Anal. Biochem. 161 (1987) 1-15, https://doi.org/10.1016/0003-2697(87)90643-9.

[32] R. Wu, E.P. Skaar, R. Zhang, G. Joachmiak, P. Gornicki, O. Schneewind, A. Joachmiak, Staphylococcus aureus IsdG and IsdI, heme-degrading enzymes with structural similarity to monooxygenases, J. Biol. Chem. 280 (2005) 28402846, https://doi.org/10.1074/ibc.M409526200.

[33] W.G. Touw, C. Baakman, J. Black, T.A.H. te Beek, E. Krieger, R.P. Joosten, G. Vriend, A series of PDB-related databanks for everyday needs, Nucleic Acids Res. 43 (2015) D364-D368, https://doi.org/10.1093/nar/gku1028.

[34] W. Kabsch, C. Sander, Dictionary of protein secondary structure: pattern recognition of hydrogen-bonded and geometrical features, Biopolymers 22 (1983) 2577-2637, https://doi.org/10.1002/bip.360221211.

[35] N. Chim, A. Iniguez, T.Q. Nguyen, C.W. Goulding, Unusual diheme conformation of the heme-degrading protein from Mycobacteriuim tuberculosis, J. Mol. Biol. 395 (2010) 595-608, https://doi.org/10.1016/j.jmb.2009.11.025.

[36] F. Neese, E.I. Solomon, MCD $C$-term Signs, Saturation Behavior, and Determination of Band Polarizations in Randomly Oriented Systems with Spin $S \geq 1 / 2$. Applications to $S=1 / 2$ and $S=5 / 2$, Inorg. Chem. 38 (1999) $1847-1865$, https://doi.org/10.1021/ic981264d.

[37] M. Gouterman, Study of the effects of substitution on the absorption spectra of porphrin, J. Chem. Phys. 30 (1959) 1139-1161, https://doi.org/10.1063/1.1730148.

[38] M.R. Cheesman, F.A. Walker, Low-temperature MCD Studies of Lowspin Ferric Complexes of Tetramesitylporphyrinate: Evidence for the Novel $\left(\mathrm{d}_{x z}, \mathrm{~d}_{y z}\right)^{4}\left(\mathrm{~d}_{x y}\right)^{1}$ Ground State which Models the Spectroscopic Properties of heme $d$, J. Am. Chem. Soc. 118 (1996) 7373-7380, https://doi.org/10.1021/JA960344I.

[39] J.A. Sigman, X. Wang, Y. Lu, Coupled oxidation of heme by myoglobin is mediated by exogenous peroxide, J. Am. Chem. Soc. 123 (2001) 6945-6946, https://doi.org/10.1021/ja015776u.

[40] A. Wilks, P.R. Ortiz de Montellano, Rat liver heme oxygenase: high level expression of a truncated soluble form and nature of the meso-hydroxylating 
species, J. Biol. Chem. 268 (1993) 22357-22362, https://doi.org/10.1016/S0021-9258(18)41536-0.

[41] R. Tenhunen, H.S. Marver, R. Schmid, Microsomal heme oxygenase: characterization of the enzyme, J. Biol. Chem. 244 (1969) 6388-6394, https://doi.org/10.1016/S0021-9258(18)63477-5.

[42] W.C. Lee, M.L. Reniere, E.P. Skaar, M.E. Murphy, Ruffling of metalloporphyrins bound to IsdG and IsdI, two heme-degrading enzymes in Staphylococcus aureus, J. Biol. Chem. 283 (2008) 30957-30963, https://doi.org/10.1074/jbc.M709486200.

[43] I. Garcia-Bosch, S.K. Sharma, K.D. Karlin, A selective stepwise heme oxygenase model system: an iron(IV)-oxo porphyrin $\pi$ cation radical leads to a verdoheme-type compound via an isoporphyrin intermediate, J. Am. Chem. Soc. 135 (2013) 16248-16251, https://doi.org/10.1021/ja405739m. 